\title{
Congestion Management under Hybrid Electricity Market using Self-organizing Hierarchical Particle Swarm Optimization
}

\author{
Manasarani Mandala \\ Electrical Engineering Department, \\ Indian Institute of Technology, Roorkee (IITR) \\ Roorkee, India
}

\author{
C.P.Gupta, PhD \\ Electrical Engineering Department, \\ Indian Institute of Technology, Roorkee (IITR), \\ Roorkee, India
}

\begin{abstract}
This paper proposes an optimal congestion management approach under hybrid electricity market using Self organizing hierarchical particle swarm optimization with Time Varying Acceleration Coefficients (SPSO-TVAC). The aim of the proposed work is to minimize deviations from preferred transaction schedules and hence the congestion cost under hybrid electricity market. The values of Transmission Congestion Distribution factors (TCDFs) are used to select redispatch of generators. Generator reactive power support is considered to lower the congestion cost. Numerical results on IEEE 57 bus system is presented for illustration purpose and the results are compared with Particle swarm optimization (PSO) in terms of solution quality. The comprehensive experimental results prove that the SPSO-TVAC is one among the challenging optimization methods which is indeed capable of obtaining higher quality solutions for the proposed problem.
\end{abstract}

\section{Keywords}

Congestion Management; Congestion cost; Transmission Congestion Distribution Factors (TCDFs); Particle Swarm optimization (PSO); Self organizing hierarchical Particle swarm optimization with Time Varying Acceleration Coefficients (SPSO-TVAC)

\section{INTRODUCTION}

The issue of transmission congestion is more pronounced in the competitive environment. Electricity markets will not be able to operate at its competitive equilibrium with congestion in the system. The main objective of congestion management is to take the actions or control measures in relieving congestion of transmission networks and increasing the power transfer capabilities. Hence transmission congestion management (TCM) plays a significant role in power system operation in deregulated environment. Several techniques of congestion management have been reported in literature [1]. In general ISO will manage the congestion management using rescheduling of generators or load curtailment. Congestion occurrence can be experienced in various forms as reported by Besharat et. al [2] which describes that the congestion can be caused by transmission line outages, generator outages, changes in demand and uncoordinated transactions. Hazra and Sinha [3] has formulated congestion management problem a bi-objective optimization problem considering alleviation of overloads and minimization of cost of congestion management as two conflicting objective functions. Talukdar et al [4], a computationally simple method is proposed for congestion management using generation rescheduling and load shed. Kumar et al. [5] have developed real and reactive power flow sensitivity factors to determine real and reactive rescheduling for congestion management using Zonal approach. Dutta and Singh [6] have used sensitivities of real power injection for rescheduling of real power generation for congestion management. Granelli et al. [7] introduced network reconfiguration is used to manage congestion to avoid costly generation or load curtailments.

Tao and Gross [8] worked on congestion relief in multilateral transaction framework, by making use of power flow over the lines to determine the contribution of individual contracts towards the congestion. Lo et al. [5] presented congestion management techniques applied to various kinds of electricity markets. Singh et al. [9], examines two approaches to dealing with management of costs. The first approach is based on pool model and the second approach is based on bilateral model. Shahidehpour et al. [10], OPF for coordination between generation companies and the ISOs for congestion management using the Benders cuts is discussed. Cutsem et al. [11], OPF is used to adjust the power injection in the least cost manner and optimal curtail transactions due to voltage instability and thermal overload. Yesuratnam and Tukkaram [12], relative electrical distance (RED) concept is introduced to mitigate the transmission overload by real power generation rescheduling. In this minimization of congestion cost is not considered. Amjady et al. [13] proposed a new congestion management framework considering dynamic voltage stability boundary of power system. Wang et al. [14], A Primal- dual Interior Point Linear Programming method is applied to solve congestion model. But these approaches have not been considered reactive power procurement. The reactive power procurement can supplement the real power to reduce the congestion cost, when the system is affected by congestion. Hence there is an urgent need for special attention to be given to reactive power procurement in congestion management of competitive electricity market. In order to operate the system in a secure manner ISO has to procure adequate amount of reactive power from various reactive resources in the system, e.g. generators, synchronous condensers and shunt capacitors. Singh et al.[16] proposed congestion management with reactive power support. Zhao et al.[15] considered the reactive power dispatch problem is a sub problem of the optimal power flow (OPF).

With the complexity of congestion management problem, an efficient heuristic approach is needed to provide the optimal solution. Different techniques have been employed to develop congestion management such as Genetic Algorithm (GA) [7], Modified benders decomposition [18], Interior Point NonLinear programming [17], Artificial Bee Colony [18], Bacterial Foraging Algorithm [19], Particle Swarm Optimization [3], Fuzzy Evolutionary programming [21]. These methods have the advantage of searching the solution space more thoroughly, but have limitations of their sensitivity to the choice of parameters such as the crossover 
and mutation probabilities, instable convergence, slow and easy to premature exist in GA, scaling factor in EP and inertia weight and learning factors in PSO. The PSO technique can generate better optimal solution in less calculation time with stable convergence characteristic compared to other population-based methods.

The PSO algorithm was introduced by Kennedy and Eberhart [22] and further modifications in PSO algorithm were carried out [23-27]. PSO is a population of random solutions, which each individual is referred as a particle and presents a candidate solution to the optimization problem. A particle in PSO like any living objects has a memory in which remain its best experience and the best experience of other particles. In this technique, each candidate solution is associated with a velocity vector, which is adjusted according to the particle's memory. This procedure is repeated until almost all particles converge to the best solution. So in each particle's point of view, its own experience and the best experience of other particles are considered and the experience of others is not regarded. Therefore the probability of becoming trapped in local minima or maxima is increased so as to be premature convergence. Kennedy and Eberhart [22] described that a relatively high value of the cognitive component, compared with the social component, will result in excessive wandering of individuals through the search space. In contrast, a relatively high value of the social component may lead particles to rush prematurely towards a local optimum. To avoid this, Wu et al. [25] proposed Particle Swarm Optimization with Time Varying Acceleration Coefficients (PSO-TVAC) would enhance convergence toward the global optima. Later, Ratnaweera et al. [26-27] proposed self organizing hierarchical particle swarm optimization with time varying particle swarm optimization (SPSO-TVAC) algorithm to enhance proper control on local optimum and global optimum. So that it can performs consistently and efficiently improves optimum solutions in the search space. So, the proposed approach considered SPSO-TVAC algorithm to manage congestion under hybrid electricity market.

Therefore the main intent of the present work is to propose a new technique Self hierarchical organizing Particle Swarm Optimization (SPSO-TVAC) for Congestion Management (CM) under hybrid electricity market. Real and reactive power Rescheduling of GENCOs' has been considered to manage congestion in the system. Real and reactive power Transmission Congestion Distribution Factors (PTCDFs \& QTCDFs) [5] are used for identifying sensitive GENCOs' for rescheduling. GENCOs' reactive power support is considered to reduce the congestion cost. The proposed algorithm SPSOTVAC has proper control on local optimum and global optimum. This algorithm performs consistently and efficiently improves optimum solutions in the search space. The proposed objective congestion cost minimization is solved using SPSO-TVAC and the obtained results are compared with PSO and PSO-TVAC.

The main contributions of this paper are as follows:

i. The proposed approach optimal congestion management under hybrid electricity market comprises of two steps. First step, step, real and reactive power Transmission Congestion Distribution Factors (PTCDFs \& QTCDFs) are used to find sensitive GENCOs'. Second step rescheduling is performed on sensitive GENCOs' based on the minimization of congestion cost under hybrid electricity market.

ii. Novel technique Self hierarchical organizing Particle Swarm Optimization (SPSO-TVAC) is used to implement the proposed approach for Congestion Management under hybrid electricity market.

\section{ADAPTIVE PARTICLE SWARM OPTIMIZATION}

\subsection{Overview of Particle Swarm Optimization (PSO)}

All PSO is a simple and efficient population-based optimization method proposed by Kennedy and Eberhart [12]. PSO consists of a swarm of particles and each particle flies through the multi-dimensional search space with a velocity, which is constantly updated by the particle's previous best performance and by the previous best performance of the particle's neighbors. The position and velocity of each particle are updated at each time step (possibly with the maximum velocity being bounded to maintain stability) until the swarm as a whole converges to an optimum. Particles update their velocity and position through tracing two kinds of 'best' value. One is its personal best (pbest), which is the location of its highest fitness value. In global version, another is the global best (gbest), which is the location of overall best value, obtained by any particles in the population. Particles update their positions and velocities according to equation (7).

$$
\begin{aligned}
V_{i d}^{K+1} & =\omega V_{i d}^{K}+c_{1} \operatorname{rand}_{1}\left(p_{i d}^{K}-x_{i d}^{K}\right)+c_{2} \operatorname{rand}_{2}\left(p_{g d}^{K}-x_{i d}^{K}\right) \\
\mathrm{x}_{i d}^{K+1} & =\mathrm{x}_{i d}^{K}+v_{i d}^{K+1}
\end{aligned}
$$

Here, $V_{\text {id }}{ }^{\mathrm{K}}$ is the velocity of $d^{\text {th }}$ dimension of the $i^{\text {th }}$ particle in the $K^{\text {th }}$ iteration, $x_{\text {id }}{ }^{\mathrm{K}}$ is the corresponding position and $p_{\text {id }}{ }^{\mathrm{K}}$ and $p_{\mathrm{gd}}{ }^{\mathrm{K}}$ is personal best and global best respectively. Finally, the position of the $i^{\text {th }}$ particle for $d^{\text {th }}$ dimension is updated by (2). Here $w$ is the inertia weight parameter which controls the global and local exploration capabilities of the particle. A large inertia weight helps in good global search while a smaller value facilitates local exploration. In order to improve the performance of the PSO, the time-varying inertia weight (PSO-TVIW) was proposed in [24].

$$
\begin{aligned}
& v_{i d}^{k+1}=C\left\{w \cdot v_{i d}^{k}+C_{1} \cdot \text { rand }_{1} \cdot\left(\text { pbest }_{i d}-X_{p d}\right)+C_{2} \cdot \text { rand }_{2} \cdot\left(\text { gbest }_{g d}-X_{i d}\right)\right\} \\
& w=\left(w_{\max }-w_{\min }\right) \cdot \frac{\left(K_{\max }-K\right)}{K_{\max }}+w_{\min } \\
& C=\frac{2}{\left|2-\varphi-\sqrt{\varphi^{2}-4 \varphi}\right|}, \quad 4.1 \leq \varphi \leq 4.2
\end{aligned}
$$

Generally for initial stages of the search process, large inertia weight to enhance the global exploration searching new area is recommended while, for last stages, the inertia weight is reduced for local exploration fine tuning the current search area. The velocity update equation is modified by the construction factor $C$ and the inertia weight $w$ is linearly decreasing as iteration grows. In The first component is the previous velocity which provides the necessary momentum for particles to roam across the problem space. The second is the cognitive component that represents the individual experience of each particle. The second component encourages the particles to move toward their own best positions reached. The last component is the social collaboration of the particles in finding the global optimal solution. The particles are pulled toward the global best particle reached. As $\varphi$ increases, the factor decreases and convergence becomes slower because population diversity is reduced.

Kennedy and Eberhart [22] described that a relatively high value of the cognitive component, compared with the social 
component, will result in excessive wandering of individuals through the search space. In contrast, a relatively high value of the social component may lead particles to rush prematurely towards a local optimum. Generally, in population-based optimization methods, it is desirable to encourage the individuals to wander through the entire search space, without clustering around local optima, during the early stages of the optimization. On the other hand, during the latter stages, it is important to enhance convergence toward the global optima, to find the optimal solution efficiently. Considering those concerns, Particle Swarm Optimization with Time Varying Acceleration Coefficients (PSO-TVAC) [25] is introduced. In PSO-TVAC method, the cognitive component is reduced and the social component is increased by changing the acceleration coefficients $\mathrm{c} 1$ and $\mathrm{c} 2$. With a large cognitive component and small social component at the beginning, particles are allowed to move around the search space, instead of moving toward the population best. On the other hand small cognitive component and large social component allows the particles to converge to the global optima in the latter part of the optimization [25]. The acceleration coefficients are expressed as,

$$
\begin{aligned}
& C_{1}=\left(C_{1 f}-C_{1 i}\right) \frac{K}{K_{\text {max }}}+C_{1 i} \\
& C_{2}=\left(C_{2 f}-C_{2 i}\right) \frac{K}{K_{\max }}+C_{2 i}
\end{aligned}
$$

where $C_{1 \mathrm{i}}, C_{1 \mathrm{f}}, C_{2 \mathrm{i}}$ and $C_{2 \mathrm{f}}$ are initial and final values of cognitive and social acceleration factors, respectively.

\subsection{Self-organizing hierarchical Particle Swarm Optimization with Time Varying Acceleration Coefficients (SPSO-TVAC)}

In this novel PSO strategy the previous velocity term in (1) is made zero. With this modification the particles rapidly rush towards a local optimum solution and then stagnate because of the absence of momentum. To make this strategy effective, the velocity vector of a particle is reinitialized with a random velocity whenever it stagnates in the search space. When a particle stagnates, its associated $p_{\text {best }}$ remains unchanged for a number of iterations. When more particles stagnate, the $g_{\text {best }}$ also undergoes the same fate and the PSO algorithm converges prematurely to a local optima and $v_{\text {id }}$ becomes zero. A necessary push to the PSO algorithm is imparted by reinitializing $v_{\text {id }}$ by a random velocity term. The method works as follows [26]:

\section{Main procedure}

Velocity update equation in (1)

if $v_{\mathrm{id}}=0$

if $\operatorname{rand} 3<0.5$

$v_{\text {id }}=$ rand $4 * v_{\text {dmax }}$

else $v_{\mathrm{id}}=-\operatorname{rand} 5{ }^{*} v_{\mathrm{dmax}}$

end if

$v_{\mathrm{id}}=\operatorname{sign}\left(v_{\mathrm{id}}\right) * \min \left(\operatorname{abs}\left(v_{\mathrm{id}}, v_{\mathrm{dmax}}\right)\right)$

Position update equation in (2)

where $v_{d \max }=\frac{\left(x_{d \text { max }}-x_{d \text { min }}\right)}{R}$,
Here $v_{\text {dmax }}$ is the maximum velocity limit on the $d^{\text {th }}$ dimension where $x_{\mathrm{dmax}}$ and $x_{\mathrm{dmin}}$ are the maximum and minimum position of particles on the $d^{\text {th }}$ dimension, respectively, and $\mathrm{R}$ is a chosen number between 1 and 10 that reflects the percentage of dynamic range of the solution on each dimension. For example, $\mathrm{R}$ could be selected as 5 for the $20 \%$ maximum velocity limit. Thus a series of particle swarm optimizers are generated inside the main PSO until the convergence criteria is reached. The variables rand $_{3}$, rand $_{4}$ and rand $_{5}$ are randomly generated numbers between 0 and 1 .

\section{RESCHEDULING OF GENCOS'}

In real time generally congestion is managed using rescheduling of GENCOs and load curtailment. In corrective action congestion management schemes, it is crucial for ISO to select the most sensitive generators to re-schedule their real and reactive powers. In the proposed approach selection of most sensitive GENCOs' for CM through sensitivity based approach using two sets of sensitivity indices, viz. Real and Reactive Power Transmission Congestion Distribution Factors (PTCDFs \& QTCDFs) for congestion management [5]. After finding sensitive GENCOs', ISO will perform rescheduling of GENCOs' based on minimization of cost using PSO, PSOTVAC and SPSO- TVAC. So, the objective is to minimize the total costs for rescheduling power. To reduce the congestion cost GENCOS' reactive power support is used. So, the proposed objective is,

Minimize $\sum_{\substack{i=1 \\ \neq s}}^{N_{G}} C_{p i}\left(\Delta P_{i}\right) \Delta P_{i}+\sum_{\substack{i=1 \\ \neq s}}^{N_{G}} C_{q i}\left(\Delta Q_{i}\right) \Delta Q_{i}$

The solution of above equation i.e., re-scheduling amount of each GENCO is obtained so that the following constraints are satisfied.

TCDFs:

$\sum_{\substack{i=1 \\ \neq s}}^{N_{G}}\left(\left(P T C D F_{i}^{k}\right) \triangle P_{i}\right)+\left(\left(Q T C D F_{i}^{k}\right) \Delta Q_{i}\right)+F_{k}^{0} \leq F_{k}^{\max } \quad k=1,2 \ldots . N_{l}$

where,$P T C D F_{j}^{k}=\Delta P_{i j} / \triangle P_{i}$

$$
Q T C D F_{j}^{k}=\Delta Q_{i j} / \Delta Q_{i} ; \quad i=1 \ldots . n_{b}, i \neq s
$$

Power flow equations: The power flow equations as determined by Kirchhoff's laws are given by,

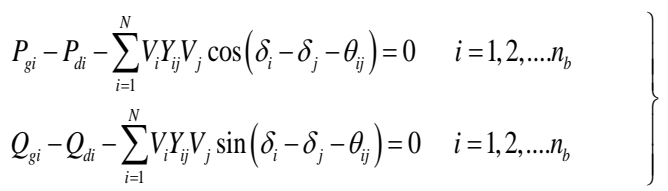

Ramp limit:

$$
\begin{aligned}
& \Delta P_{i}^{\min } \leq \Delta P_{i} \leq \Delta P_{i}^{\max } \quad i=1,2 \ldots N_{g}, i \neq s \\
& \Delta Q_{i}^{\min } \leq \Delta Q_{i} \leq \Delta Q_{i}^{\max } \quad i=1,2 \ldots N_{g}, i \neq s
\end{aligned}
$$

Power limit of generation:

$$
\begin{array}{ll}
P_{i}^{\min } \leq P_{i}+\Delta P_{i} \leq P_{i}^{\max } & i=1,2 \ldots . N_{G}, i \neq s \\
Q_{i}^{\min } \leq P_{i}+\Delta P_{i} \leq Q_{i}^{\max } & i=1,2 \ldots . N_{G}, i \neq s \\
V_{i}^{0}-V_{i}^{\min } \leq \Delta V_{i} \leq V_{i}^{\max }-V_{i}^{0} & i=1 \ldots \ldots . .
\end{array}
$$

Power balance constraint: 
$P_{G m}-P_{G n}=0, Q_{G m}-Q_{G n}=0$

$\left.\sum_{m} P_{G m}^{t}-\sum_{n} P_{D n}^{t}=0, \sum_{m} Q_{G m}^{t}-\sum_{n} Q_{D n}^{t}=0, \quad t=1,2 \ldots N_{t}\right\}$

Transmission line limits: Transmission line flows are bounded by thermal limits for short lines and stability limits for long lines.

$$
S_{l} \leq S_{l}^{\max } \quad l=1,2, \ldots \ldots N_{L}
$$

where, PGm and QGm stand for the real and reactive power transactions into the seller bus-m, respectively and PDn and QDn are the real and reactive powers taken out at the buyer bus-n, respectively and t represents the total number of such transactions. ' $t$ ' represents bilateral/multilateral transaction.

\section{Rescheduling Cost:}

Here Rescheduling cost is considering both Fuel cost coefficients and emission coefficients.

$$
C_{p i}\left(\Delta P_{i}\right)=F\left(\Delta P_{i}\right)+E\left(\Delta P_{i}\right)
$$

Minimization of Fuel Cost:

$$
F\left(\Delta P_{i}\right)=a_{i}\left(\Delta P_{i}\right)^{2}+b_{i}\left(\Delta P_{i}\right)+C_{i}
$$

$\mathrm{C}_{\mathrm{pi}}$ is the cost of the active power rescheduling according to the bid functions submitted by the generators participating in congestion management.

\section{Minimization of Emission:}

The total emission $E\left(\Delta P_{i}\right)$ in (ton/hr) of atmospheric pollutants such as sulpher oxides $\left(\mathrm{SO}_{\mathrm{X}}\right)$ and nitrogen Oxides $\left(\mathrm{NO}_{\mathrm{X}}\right)$ caused by the operation of fossil fuelled thermal generation can be expressed as,

$$
E\left(\Delta P_{i}\right)=\sum_{i=1}^{N} 10^{-2}\left(\alpha_{i}+\beta_{i}\left(\Delta P_{i}\right)+\gamma_{i}\left(\Delta P_{i}^{2}\right)\right)+\varsigma_{i} \exp \left(\lambda_{i}\left(\Delta P_{i}\right)\right)
$$

Where $\alpha_{i}, \beta_{i}, \varsigma_{i}$ and $\lambda_{i}$ are coefficients of the $\mathrm{i}^{\text {th }}$ generator emission characteristics.

Generator Reactive Power support:

$$
C_{q i}\left(\Delta Q_{i}\right)=\left[C_{p i}\left(S_{G i \max }\right)-C_{p i}\left(\sqrt{S_{G i \max }^{2}-\Delta Q_{i}^{2}}\right] k_{i}\right.
$$

Where, $\mathrm{C}_{\mathrm{qi}}$ is the cost of the Reactive power rescheduling, $\mathrm{C}_{\mathrm{pi}}$ is the active power generation cost, $\mathrm{S}_{\mathrm{G} \text { max }}$ is the nominal apparent power of the generator and $\mathrm{k}_{\mathrm{i}}$ is an assumed profit rate of the active power generation at bus i. Here $k_{\mathrm{i}}$ is taken as $5 \%$. Here $S_{\text {Gimax }}=P_{\text {Gimax }}$

As mentioned in section II, the proposed objective is solved using PSO, PSO-TVAC and SPSO-TVAC. To now the efficiency of proposed approach is applied on IEEE- 30 bus and IEEE- 57 bus system. So, next section is dealing with results and discussion.

\section{RESULTS AND DISCUSSION}

As mentioned in section I, the proposed problem comprising of two steps. First step, sensitive GENCOs' are selected for CM through sensitivity based approach using two sets of sensitivity indices, viz. PTCDFs \& QTCDFs. Second step, based on the available bids ISO will perform rescheduling of GENCOs' based on minimization of cost using PSO, PSOTVAC and SPSO- TVAC. To illustrate the efficiency of the proposed idea for congestion Management is applied on IEEE- 57 bus system. The parameters used for PSO, PSO-
International Journal of Computer Applications (0975 - 8887)

\begin{tabular}{|c|c|c|c|c|}
\hline Parameters & CPSO & PSO-TVIW & PSO-TVAC & SPSO-TVAC \\
\hline \multirow{2}{*}{$\mathrm{C} 1$} & \multirow{2}{*}{2} & \multirow{2}{*}{2} & $\mathrm{C}_{1 \mathrm{i}}=2.5$ & $\mathrm{C}_{1 \mathrm{i}}=2.5$ \\
\hline & & & $\mathrm{C}_{1 \mathrm{~F}}=0.2$ & $\mathrm{C}_{1 \mathrm{~F}}=0.2$ \\
\hline \multirow{2}{*}{$\mathrm{C} 2$} & \multirow{2}{*}{2} & \multirow{2}{*}{2} & $\mathrm{C}_{2 \mathrm{i}}=2.5$ & $\mathrm{C}_{2 \mathrm{i}}=2.5$ \\
\hline & & & $\mathrm{C}_{2 \mathrm{~F}}=0.2$ & $\mathrm{C}_{2 \mathrm{~F}}=0.2$ \\
\hline \multirow{2}{*}{ W } & \multirow{2}{*}{0.5} & $\mathrm{~W}_{\min }=0.4$ & $\mathrm{~W}_{\min }=0.4$ & \multirow{2}{*}{0} \\
\hline & & $\mathrm{W}_{\max }=0.9$ & $\mathrm{~W}_{\mathrm{MAX}}=0.9$ & \\
\hline $\mathrm{C}$ & -- & $\varphi=4.1$ & $\varphi=4.1$ & -- \\
\hline $\begin{array}{l}\text { Computation } \\
\text { time (Secs) }\end{array}$ & 121.43 & 119.66 & 103.45 & 89.76 \\
\hline $\begin{array}{l}\text { No. of } \\
\text { iterations }\end{array}$ & 60 & 60 & 50 & 50 \\
\hline
\end{tabular}

Volume 82 - No 17, November 2013

TVAC and SPSO- TVAC to solve the proposed problem are given in Table I.

Table I: Parameters variation for all techniques

\section{IEEE-57 bus system:}

The proposed approach congestion management under hybrid electricity market using SPSO-TVAC is tested on IEEE-57 bus system. The numerical data for IEEE-57 bus system are taken from [27]. It consists of seven GENCOs' and eighty transmission lines. To analyze the proposed approach different combinations of market structures comprising pool model and mix of pool plus bilateral and multilateral contracts are considered and listed in Table II.

Table II: Bilateral/ Multilateral transactions in IEEE-57 bus system

\begin{tabular}{|c|c|c|c|}
\hline Transactions & From & To & Power (MW) \\
\hline T1 (Bilateral) & 7 & 33 & 12 \\
\hline \multirow{2}{*}{ T2 (Bilateral) } & 11 & 24 & 12 \\
\cline { 2 - 3 } & 7 & 33 & 10 \\
\hline $\begin{array}{c}\text { T3 } \\
\text { (Multilateral) }\end{array}$ & 7 & 33 & 16 \\
\cline { 3 - 3 } $\begin{array}{c}\text { T4 } \\
\text { (bilateral \& } \\
\text { Multilateral) }\end{array}$ & 7 & 26 & \multicolumn{2}{|c}{14} \\
\cline { 2 - 3 } & 71 & 24 & \\
\end{tabular}

Because of these contracts congestion occurred between 3-4 \& 6-9 lines. The congested line details are given in Table III.

Table III: Congested line details for IEEE-57bus system

\begin{tabular}{|c|c|c|c|c|}
\hline \multirow{2}{*}{$\begin{array}{c}\text { Congested } \\
\text { lines }\end{array}$} & \multicolumn{3}{|c|}{ Power flow in lines } & \multirow{2}{*}{$\begin{array}{c}\text { Line limit } \\
\text { (MW) }\end{array}$} \\
\hline $6-7$ & 114.97 & 113.53 & $\begin{array}{c}\text { SPSO- } \\
\text { TVAC }\end{array}$ & 112.33 \\
\hline $14-15$ & 163.77 & 162.45 & 160.03 & 100 \\
\hline
\end{tabular}

To perform CM firstly sensitive GENCOs' are selected based on PTCDFs and QTCDFs. In this system, PTCDFs and QTCDFs are computed for the congested lines 6-7 and 14-15 and depicted in Fig. 1 (a) \& (b). 


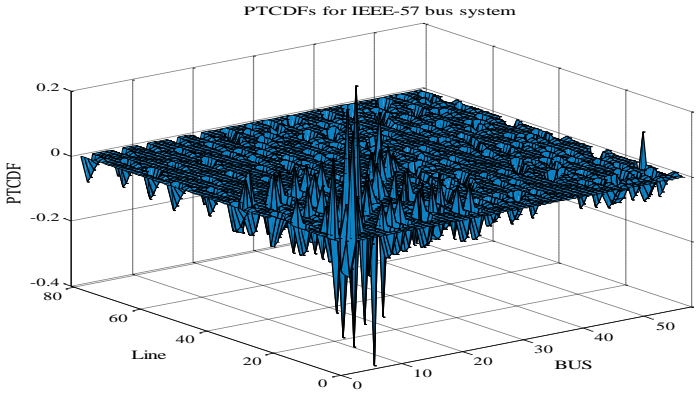

Fig.1 (a). PTCDFs for IEEE- 57 bus system



Fig.1 (b) QTCDFs for IEEE- 57 bus system

Table IV: Rescheduling results for IEEE-57 bus system

\begin{tabular}{|c|c|c|c|c|c|c|}
\hline & \multicolumn{6}{|c|}{ Active Power Re-scheduling } \\
\hline Genco & Technique & $\mathrm{P}$ & T1 & $\mathrm{T} 2$ & T3 & $\mathrm{T} 4$ \\
\hline \multirow{3}{*}{ G2 } & $\mathrm{PSO}$ & +4.44 & +2.21 & +2.45 & +2.97 & +3.31 \\
\hline & PSO-TVAC & +3.59 & +2.03 & +2.25 & +2.73 & +3.03 \\
\hline & SPSO-TVAC & +3.03 & +1.80 & +1.99 & +2.43 & +2.70 \\
\hline \multirow{3}{*}{ G12 } & PSO & +11.33 & +7.96 & +8.60 & +9.11 & +10.28 \\
\hline & PSO-TVAC & +10.15 & +6.55 & +8.04 & +8.34 & +9.48 \\
\hline & SPSO-TVAC & +9.36 & +6.03 & +6.53 & +7.63 & +8.72 \\
\hline \multirow{3}{*}{ G9 } & PSO & -8.49 & -5.81 & -6.67 & -6.99 & -7.34 \\
\hline & PSO-TVAC & -7.32 & -5.24 & -5.96 & -6.34 & -6.93 \\
\hline & SPSO-TVAC & -5.95 & -3.62 & -4.08 & -4.54 & -5.16 \\
\hline \multirow{3}{*}{ G3 } & $\mathrm{PSO}$ & -6.68 & -3.93 & -4.59 & -5.38 & -5.83 \\
\hline & PSO-TV & -5.91 & 56 & -4.26 & -4.56 & -5.07 \\
\hline & SPSO-TVAC & -4.17 & -2.37 & -2.93 & -3.29 & -3.67 \\
\hline & \multicolumn{6}{|c|}{ Reactive power Re-scheduling } \\
\hline Genco & Technique & $\mathrm{P}$ & $\mathrm{C} 1$ & $\mathrm{C} 2$ & $\mathrm{C} 3$ & $\mathrm{C} 4$ \\
\hline \multirow{3}{*}{ G3 } & $\mathrm{PSO}$ & +3.70 & +1.80 & +2.14 & +2.66 & +3.06 \\
\hline & PSO-TVAC & +3.13 & +1.30 & +1.83 & +2.23 & +2.60 \\
\hline & SPSO-TVAC & +2.88 & 73 & +1.94 & +2.31 & +2.67 \\
\hline \multirow{3}{*}{ G9 } & PS & +4.16 & 07 & +2.76 & +2.97 & +3.48 \\
\hline & PSO-TVAC & +3.36 & +1.90 & $=2.11$ & +2.55 & +2.84 \\
\hline & SPSO-TVAC & +2.84 & +1.68 & +1.86 & +2.28 & +2.52 \\
\hline \multirow{3}{*}{ G2 } & PSO & -1.75 & -0.99 & -1.19 & -1.35 & -1.56 \\
\hline & PSO-TVAC & -1.68 & -0.94 & -1.14 & -1.29 & -1.48 \\
\hline & SPSO-TVAC & -1.58 & -0.90 & -1.07 & -1.22 & -1.39 \\
\hline \multirow{3}{*}{ G12 } & PSO & -6.48 & -4.95 & -5.33 & -5.54 & -5.91 \\
\hline & PSO-TV & -5.58 & -4.55 & -4.30 & -4.81 & -5.07 \\
\hline & SPSO-TVAC & -4.64 & -3.00 & -3.57 & -3.86 & -4.29 \\
\hline
\end{tabular}

From the Fig. 1(a) it is observed that GENCOs' G2, G12, G9 \& G3 are having large PTCDFs and are selected for real power rescheduling. Similarly, From the Fig. 1(b) it is observed that GENCOs' G3, G9, G2 \& G12 are having large QTCDFs and are selected for reactive power rescheduling. After selecting sensitive GENCOs' ISO will perform GENCOs' rescheduling based on minimization of cost using PSO, PSO-TVAC and SPSO-TVAC and the amount of rescheduling is listed in Table IV. From the Table IV, it is observed that GENCOs' G2 and G12 are increased real power and G8 and G3 are decreased real power to manage congestion. Similarly, GENCOs' G3 and G9 are increased reactive power and G2 and G12 are decreased reactive power to manage congestion in the system. Under pool transaction, GENCO G2 increased real power 4.44 MW using PSO and it is reduced to $3.59 \mathrm{MW}$ using PSO-TVAC and 3.03 MW using SPSO-TVAC. Similarly under all transactions real and reactive power rescheduling amount is reduced using SPSOTVAC than PSO and PSO-TVAC. So, SPSO-TVAC is performed better than PSO and PSO-TVAC for the objective minimization of GENCOs' rescheduling amount, hence congestion cost. Based on the real and reactive power rescheduling congestion cost is computed and listed in Table V.

Table V: Cost details for IEEE-57 bus system

\begin{tabular}{|c|c|c|c|c|c|c|}
\hline (\$/hr) & Techniques & Pool & T1 & T2 & T3 & T4 \\
\hline \multirow{2}{*}{$\begin{array}{c}\text { Congestion } \\
\text { cost }\end{array}$} & PSO & 875.61 & 683.42 & 721.65 & 769.11 & 810.24 \\
\cline { 2 - 7 } & PSO-TVAC & 788.68 & 610.53 & 650.16 & 691.15 & 719.79 \\
\cline { 2 - 7 } & PSO-TVAC & 692.53 & 529.98 & 570.76 & 617.13 & 650.82 \\
\hline $\begin{array}{c}\text { During CM } \\
\text { GENCOs' } \\
\text { cost }\end{array}$ & PSO & 3044.0 & 2506.6 & 2655.4 & 2741.5 & 2896.7 \\
\cline { 2 - 7 } & PSO-TVAC & 2826.1 & 2448.5 & 2586.8 & 2659.5 & 2796.4 \\
\hline $\begin{array}{c}\text { After CM } \\
\text { GENCOs' } \\
\text { cost }\end{array}$ & PSO & 3221.8 & 2335.4 & 2493.0 & 2689.9 & 2960.0 \\
\cline { 2 - 7 } & PSO-TVAC & 3121.9 & 2263.0 & 2415.7 & 2606.6 & 2868.2 \\
\cline { 2 - 7 } & SPSO-TVAC & 3024.8 & 2192.7 & 2340.6 & 2525.5 & 2779.1 \\
\hline
\end{tabular}

From the Table $\mathrm{V}$, it is observed that congestion cost under pool transaction is $875.61(\$ / \mathrm{hr})$ using PSO and it is reduced to $788.68(\$ / \mathrm{hr})$ using PSO-TVAC and 692.53(\$/hr) using SPSO-TVAC. Similarly for all transactions congestion cost is reduced using SPSO-TVAC than PSO and PSO-TVAC. For the comparison of congestion under all transactions using PSO, PSO-TVAC and SPSO-TVAC is depicted in Fig. 2. From the Fig. 2 it is cleared that congestion cost is reduced using SPSO-TVAC under all transactions. So, SPSO-TVAC is performed better than PSO and PSO-TVAC to solve the objective of congestion cost minimization.

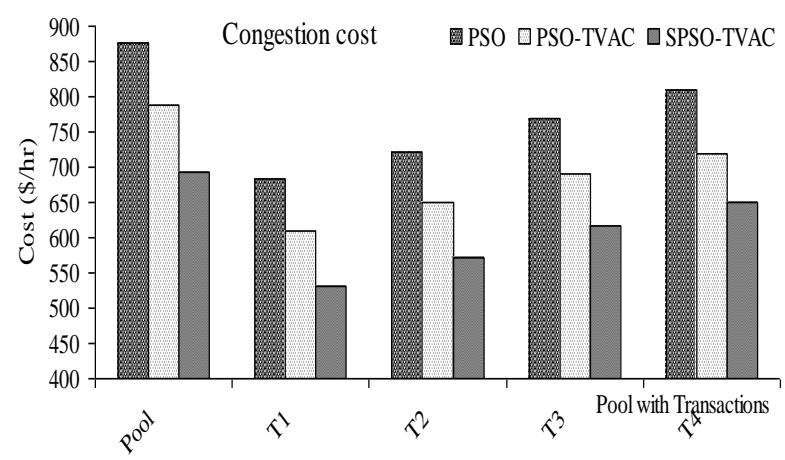

Fig.2.Congestion cost details for IEEE- 57 bus system

The congestion cost convergence criterion under pool transaction using PSO, PSO-TVAC and SPSO-TVAC is depicted in Fig.6. From the Fig.6, it is observed that PSO finds better solution after 76 iterations, PSO-TVAC finds better solution after 57 iterations and SPSO-TVAC finds better solution after 51 iterations. So, SPSO-TVAC is performed better than PSO-PSO-TVAC and SPSO-TVAC, hence convergence time. 


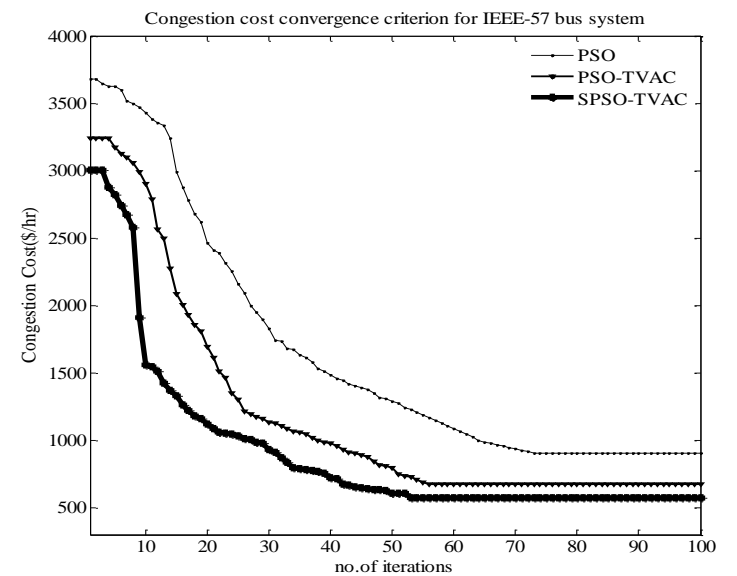

Fig.3. Congestion cost convergence criterion for IEEE- 57 bus system

After performing CM, the power flow in the congested line is listed in Table VI.

Table VI: Congested line details after CM for IEEE-57 bus system

\begin{tabular}{|c|c|c|c|c|}
\hline \multirow{2}{*}{$\begin{array}{c}\text { Congested } \\
\text { lines }\end{array}$} & \multicolumn{3}{|c|}{ Power flow in lines } & Line limit \\
\cline { 2 - 4 } & PSO & PSO-TVAC & SPSO- TVAC & $(\mathrm{MW})$ \\
\hline $6-7$ & 99.44 & 98.66 & 98.02 & 100 \\
\hline $14-15$ & 149.47 & 148.85 & 148.21 & 150 \\
\hline
\end{tabular}

After performing $\mathrm{CM}$, based on the available bids and power dispatch GENCOs' cost is computed and compared with GENCOs' cost during CM is listed in Table V. From the Table $\mathrm{V}$, it is observed that under pool transaction during $\mathrm{CM}$ using PSO is $3044.0(\$ / \mathrm{hr})$ and it is increased to $3221.8(\$ / \mathrm{hr})$. Similarly, for all transaction GENCOs' cost is increased after CM. under pool transaction GENCOs' cost after CM using PSO is $3221.8(\$ / \mathrm{hr})$ it is reduced to $3121.9(\$ / \mathrm{hr})$ using PSOTVAC and 3024.8(\$/hr) using SPSO-TVAC. Similarly for all transaction during and after CM using SPSO-TVAC GENCOs' cost is lesser than PSO and PSO-TVAC. From the obtained results, SPSO-TVAC is performed better for congestion management using rescheduling of GENCOs' under hybrid electricity market.

\section{CONCLUSION}

This paper focuses on congestion management under hybrid electricity market using self organizing hierarchical particle swarm optimization with time varying particle swarm optimization (SPSO-TVAC). The proposed algorithm has proper control on local optimum and global optimum, so that it can performs consistently and efficiently improves optimum solutions in the search space. The proposed approach is considered to manage congestion in the system by GENCOs' real and reactive rescheduling. GENCOs' reactive power support is helping to reduce the congestion cost. After Congestion Management total GENCOs' cost is increased for all transactions. From the results obtained demonstrate the performance of the proposed approach transmission congestion under hybrid electricity market based SPSOTVAC in term of solution quality and convergence characteristic.

\section{REFERENCES}

[1] Sood, Y.R., Padhy, N.P., Gupta, H.O., "Wheeling of power under deregulated environment of power system a bibliographical survey", IEEE Trans. Power Syst., Vol.17, pp.870-8, Aug 2002.

[2] Besharat, H., and Taher, S.A. 2008. Congestion management by determining optimal location of TCSC in deregulated power systems. Int. Journal. Elect. Power Energy Syst. Vol.30, pp. 563-568

[3] Hazra, J., and Sinha, A.K. 2007. Congestion management using multi objective particle swarm optimization. IEEE Trans. Power Syst., Vol.22, pp.17261734.

[4] Talukdar, B. K., Sinha .A. K., Mukhopadhyay, S., and Bose. A. 2005. A computationally simple method for cost- efficient generation rescheduling and load shedding for congestion management. Int. Journal. Elect. Power Energy Syst., Vol.27, pp. 379-388.

[5] Ashwani Kumar, Srivastava, S.C., and Singh, S.N. 2004. A Zonal Congestion Management Approach Using Real and Reactive Power Rescheduling. IEEE Trans. Power Syst., Vol. 19, pp. 554-561.

[6] Sudipta Dutta., and Singh, S. P. 2008. Optimal Rescheduling of Generators for Congestion Management Based on Particle Swarm Optimization. IEEE Trans. Power Syst., Vol. 23, pp. 1560-1568.

[7] Granelli, G., Montagna, M., Zanellini, F., Bresesti, P., Vailati, R., and Innorta, M. 2006. Optimal network reconfiguration for congestion management by deterministic and genetic algorithms. Int. Journal. Elect. Power Syst. Res. Vol.76, pp. 549-556.

[8] Tao, S., and Gross, G. 2002. A congestion management allocation mechanism for multiple transaction networks. IEEE Trans. Power Syst., vol.17, pp.826-833.

[9] Lo, K.L., Yuen, Y.S., and Snider, L.A. 2000. Congestion management in deregulated electricity markets, in: IEEE International Conference on Electric Utility Deregulation and Restructuring and Power Technologies 2000, pp. 4752 .

[10] Singh, H., Hao, S., and Papalexopolous, A. 1998. Transmission congestion management in competitive electricity markets. IEEE Trans. Power Syst., Vol.13, pp.672-680.

[11] Yamina, H.Y., and Shahidehpour, S.M. 2003. Congestion management coordination in the deregulated power market. Int. Journal. Elect. Power Syst. Res., vol. 65, pp. 119-127.

[12] Capitanescu, F., and Cutsem, T.V. 2007. A unified management of congestions due to voltage instability and thermal overloads. Int. Journal. Elect. Power Syst. Res. Vol. 77, pp.1274-1283.

[13] Yesuratnam, G., and Thukaram, D. 2007. Congestion management in open access based on relative electrical distances using voltage stability criteria. Int. Journal. Elect. Power Syst. Res. Vol .77, pp.1608-1618.

[14] Amjady, N., and Hakimi, M. 2012. Dynamic voltage stability constrained congestion management framework for deregulated electricity markets. Int. Journal. Energy Conv. and Management. Vol.58, pp. 66-75

[15] Zhao, B., Guo, C.X., and Cao, Y.J. 2005. A multi-agent based particle swarm optimization approach for reactive power dispatch. IEEE Trans. Power Syst. Vol.20, pp.1070-78.

[16] Kanwardeep Singh, Padhy, N. P., and Sharma, J. D. 2009. Social Welfare Maximization Considering 
Reactive Power and Congestion Management in the Deregulated Environment. Vol.38. pp.50-71.

[17] Wang, X., Song, Y H., and Lu, Q. 2000. Primal-dual Interior Point Linear Programming Optimal Power Flow for Real-time Congestion Management. IEEE Power Engg. Society Winter Meeting, Singapore. pp1643 1649.

[18] Masoud, E., Ebadi, F., Ali Shayanfar, H., and Jadid, S. 2013. Congestion management in hybrid power markets using modified Benders decomposition. Applied Energy. Vol.102, pp.1004-1012.

[19] Jain, A. K., Srivastava, S. C., Singh, S. N., and Srivastava, L. 2012. Strategic Bidding in Transmission Constrained Electricity Markets Using Artificial Bee Colony Algorithm. Electric Power Components and Syst. Vol.40, pp.1768-88.

[20] Panigrahi, B.K., and Ravikumar Pandi, V. 2009. Congestion management using adaptive bacterial foraging algorithm. Int. Journal. Energy Conv. and Management. and Management. Vol. 50, pp.1202-1209.

[21] Vijayakumar, K. 2012.Multiobjective Optimization Methods for Congestion Management in Deregulated Power Systems. Jour. of Elect. and Computer Engg. Vol. 2012.
[22] Kennedy, J., and Eberhart, R. 1995. Particle swarm optimization", Proceedings of the IEEE International Conference on Neural Networks. pp. 1942- 1948.

[23] Shi, Y., and Eberhart, R.C. 1999. Empirical study of particle swarm optimization. Proc. of the Int. Congress on Evolutionary Computation, Vol. 3, pp.101-106.

[24] Eberhart, R. C., and Shi, Y. 2000. Comparing inertia weights and constriction factors in particle swarm optimization. in Proc. Congr. Evolutionary Computation, Vol. 1, pp. 84-88.

[25] He, S., Wu, Q. H., Wen, J. Y., Saunders, J. R., and Patton, P. C. 2004. A particle swarm optimizer with passive congregation. Biosystems, Vol. 78, pp. 135-147.

[26] Ratnaweera, A. , Halgamuge, S. K., and Watson, H. C. 2004. Self-organizing hierarchical particle swarm optimizer with time-varying acceleration coefficients. IEEE Trans. Evol. Comput., Vol. 8, pp. 240-255.

[27] Chaturvedi, K. T., Pandit, M., and Srivastava, L. 2008. Self-organizing hierarchical particle swarm optimization for non-convex economic dispatch. IEEE Trans. Power Syst. Vol. 23, pp. 1079-1087.

[28] http://www.ee.washington.edu/research/pstca/ 